\title{
ESPACIOS DE APRENDIZAJE PARA EL DESARROLLO DE LA INTELIGENCIA COLECTIVA
}

\author{
Betty Monroy Henao* \\ Dpto. de Tecnología, Facultad de Ciencia y Tecnología, Universidad \\ Pedagógica Nacional.
}

\begin{abstract}
Exponential acceleration of production and transmission of information fea tures current society, development celerity and diversification of technological tools for information processing and storage make an increasing amount of elaborate knowledge and data available for citizens on the entire world around. This article sustains the thesis that this phenomenon, with no antecessor, generates a kind of group intelligence which should be improved by means of two strong strategies:

design and implement social and cultural settings for understanding andproducing shared information, and incorporate technological tools in order to empower those settings.
\end{abstract}

\section{RESUMEN}

Una característica del mundo contemporáneo es el aumento exponencial del volumen de información que diariamente se produce y transmite en el mundo. La celeridad del desarrollo y a diversificación de los soportes de la información ponen al alcance de muchos ciudadanos un gran volumen de información y conocimiento elaborado. El articulo trata de plantear que este hecho sin precedentes en la humanidad, genera una suerte de inteligencia colectiva que hay que potenciar a través de dos estrategias fuertes:

— creación de espacios culturales colectivos de comprensión y producción de información compartida, $\mathrm{y}$,

— empleo de medios tecnológicos que amplifiquen tales espacios.

\section{INTELIGENCIA COLECTIVA}

La afirmación de que estamos en la era de la información tiene cada vez más sentido en nuestras sociedades. La información que comparte un grupo social exige elementos que sirvan de puente entre los individuos. Sin duda el lenguaje ha cumplido esta función histórica. Contando por supuesto con las funciones neurológicas asociadas, el lenguaje convierte la información en un bien colectivo y a su vez crea nueva información de orden cultural. Empleando el símil de que Los genes propagan un acervo de información genética a través de las células, la información se propaga hoy a través de las nodos (personas) y las redes (conjuntos intercomunicados de personas), sociales y electrónicas. La información se propaga cuando salta' de un cerebro a otro. Fundamentalmente la 
información es variedad y es riqueza cultural. El proceso de socialización humana genera entre las personas nexos importantes, pues ninguna de ellas llega a tener la totalidad de la información. De alguna manera, todos dependemos de la parte de información que guarda el otro.

A decir de Lévy (1997:253), "la interconexión entre los individuos fomenta procesas de inteligencia colectiva en sociedades virtuales donde los individuos están menas protegidos frente al caos de la información. Idealmente, la movilización de Las tecnologías de la información crea una suerte de inteligencia colectiva que significa potenciación de la misma, uso y sinergia de capacidades individuales y diversidad en formas y lugares para encontrarla y producirla".

Inteligencia colectiva significa entonces, distribución y producción colectiva de conocimiento, aprendizaje compartido, creación de redes de información, desarrollo de capacidades cognitivas a partir del enorme potencial humano resultante de la combinación de memoria e inteligencia, uso de recursos y habilidades, sinergia de proyectos y posibilidad del estímulo mutuo a nuestras similitudes y a nuestras diferencias.

El empleo de tecnologías digitales y de redes de comunicación interactiva en la vida de las personas y los grupos, potencialmente generará un cambio cultural situado en tres aspectos: en las relaciones sociales, en el conocimiento y en nuestras capacidades cognitivas. Se considera así que las tecnologías (inteligentes) van a redefinir el alcance, el sentido y eventualmente, la naturaleza de las potencialidades humanas como la memoria, la percepción, el razonamiento y la imaginación.

Se abre así paso a criterios temporales y contextuales, de eficiencia, de capacidad para solucionar problemas y de cambio. La universalidad se da más concretamente en la capacidad de conectarse e interactuar entre las personas y los grupos humanos. El ciberespacio es la infraestructura para producir, conectar y manejar información y saber. En pacas décadas el ciberespacio será el medio primordial para la inteligencia colectiva de la humanidad.

Eventualmente, las nuevas tecnologías conducen al funcionamiento de grupos humanos que usen su inteligencia colectiva pera esta no ocurre automáticamente. La renuencia a comprender su poder, la inflexibilidad de las instituciones, la inercia de las mentalidades o de la cultura pueden llevar a que se desconozca el uso social de la información, potenciado con el empleo de las nuevas tecnologías.

La inteligencia colectiva es un tipo de "corporación"1 en la cual cada participante aporta como capital su conocimiento, su experiencia y su habilidad para aprender y enseñar. Exalta las capacidades individuales para que se desarrollen en interacción con otros. La hipótesis que subyace, es que es posible y deseable construir social y técnicamente significados que se materialicen en una inteligencia colectiva y que se potencie a través del emplea de grandes redes digitales de información, memorias de computadoras e interfaces interactivas multimodales.

Compartir información y generar conocimiento colectivo conlleva diseñar una nueva arquitectura en el ciberespacio. Los nuevos arquitectos pueden ser ingenieros, diseñadores de interfaces a de redes, programadores de software, organizaciones

\footnotetext{
${ }^{1}$ Nombre genérico con el cual Lévy (1997) designa grupos o personas que navegan en el ciberespacio y comparten información entre sí.
} 
nacionales e internacionales, artistas, educadores que integran lo pedagógica y lo tecnológico y en general personas con educación y experiencia que consideren las impactas políticos, económicos y culturales de estas alternativas tecnológicas.

Los diseñadores de ciberespacios podrán ayudar a producir ambientes simbólicos que desarrollen operaciones intelectuales (sistemas de signos, lenguajes, programas, competencias específicas), propicien acciones (telecomunicaciones, operaciones remotas) y potencien la comunicación en muchas sociedades y culturas diversas en términos de la globalidad del planeta.

Diseñar el ciberespacio es una tarea contemporánea para muchas personas y grupos cada vez más amplios envueltas en el procesamiento y producción de información. En la medida en que se multiplique la posibilidad de compartir y generar información por diferentes sectores de las saciedades se tendrá el reto de diseñar ambientes propicios para ella. En este sentido, tales ambientes pueden contener:

- Instrumentos que promuevan el desarrollo de vínculos de aprendizaje e intercambio de conocimiento.

- Métodos de comunicación dispuestos para reconocer, integrar y renovar la diversidad de información.

- Sistemas de navegación y procesamiento que promuevan comportamientos autónomos.

- Sistemas de ingeniería semiótica capaces de procesar datos, información, competencias y el gran poder simbólico acumulado por la humanidad.

Resulta fácil prever un creciente éxito de las nuevas formas de "corporaciones" en el marco de una inteligencia colectiva. Primero en redes informáticas clásicas, como en las redes que permiten el intercambio de correo electrónico (Internet, Arpanet, CSN EI, SPAN, MAILNET en los Estados Unidos, JANET en Gran Bretaña, COSAC en Francia, DFN en Alemania) o en las redes cooperativas cama BITNET en Estados Unidos, EARN en Europa a Asianet en Japón. La etapa actual es aprovechar las capacidades de las redes digitales con integración de servicias como Númeris para transportar información en tiempo real y animar la interacción entre agentes (Quéau, 1995: 69-70).

En el fondo, la inteligencia colectiva busca asumir una preocupación y darle salida: la interacción entre agentes (autónomos) se puede volver muy compleja rápidamente porque su evolución es continua y puede llevar a una gran anarquía en una red. Una anarquía demasiado radical puede socavar los mismos fundamentos que la hacen pasible. Una forma de regulación es estimular nuevas formas de percepción y comprensión del entorno de la información y asumir que hay riesgos. Las comunidades que se forman en las redes son comunidades sociales y los individuos que las componen establecen lazos afectivos y cognitivos que intercambian entre si y se colaboran. La gente hace relaciones reales aunque se haga una nueva distribución de los ámbitos públicos y privados con el desarrollo de las redes y las ciberculturas.

\section{ALGUNAS CONSIDERACIONES E DUCATIVAS}

Crear espacias de desarrollo para la inteligencia colectiva requiere considerar algunos aspectos contemporáneos con fuertes implicaciones educativas:

a. El entorno en el cual nos desenvolvemos, implica cada vez más das retos básicos: aprender a procesar y producir información y crear ambientes que promuevan el 
multiculturalismo y la comprensión de la interdependencia de los sistemas en el mundo de hoy (Tye, 1991: 18). Este planteamiento conlleva a que las personas se desenvuelvan en el mundo de las tecnologías de la información y la comunicación (TIC) y a que se utilicen para proporcionar una mejor educación tanto en lo personal como en las comunidades nacionales e internacionales. El emplea de las TIC en los centros educativos permite el trabajo colaborativo entre estudiantes de cualquier parte del planeta y la educación en la multiculturalidad permite comprender a otros, entender problemas sociales y apreciar las diferencias culturales entre los pueblos.

b. De otra parte, es preciso plantearse el problema de la educación global. Este concepto conlleva enormes complejidades sociales y culturales. La sociedad global se viene implantando como un horizonte de interacción planetaria y universal. Este aspecto es fascinante por unos lados y angustiante por otras. En educación por ejemplo, pueden crearse espacios donde se compartan valores, lenguajes, costumbres, saberes, proyectos, todo ello de enorme diversidad. Por otro lado, la sociedad global pide a los individuos un gran esfuerzo de orientación y de identidad personal. En un mundo de interacciones y grandes diferencias compartidas se requiere una educación que potencie a las personas para no perderse en tanta información y para estructurar competencias sociales, cognitivas y valorativas propias de un mundo tan complejo y a la vez tan diverso.

La globalidad puede describirse en los planos material, social y temporal (Scheunpflug, 1997: 79). En todos los aspectos, en los países industrializados y los llamados en desarrollo, tienen nuevos desafíos. Los individuos de hoy tienen que aprender a manejarse ante situaciones de gran complejidad y experiencias divergentes en muchos planos. Se vive hay una situación histórica en la cual la rapidez de los cambios sociales rebasa los cambios personales. Nuestra capacidad para resolver problemas puede ir a la zaga de la evolución del mundo, sino nos preparamos para los nuevos retos del mundo en un futuro muy próximo.

Se puede afirmar que el pensamiento, el lenguaje, la ciencia y la tecnología nos ponen en condiciones de salir de nuestros microcosmos y ponernos en contacto con la sociedad global. La cognición nos permite afrontar y resolver problemas complejos y la educación en diferentes procesas cognitivas, culturales, científicas y tecnológicos nos permitirá asumir competencias básicas para los nuevos retos:

— Orientación hacia solución de problemas.

- Autoaprendizaje y autorregulación

- Toma de decisiones y métodos de planteamiento de preguntas.

- Capacidad para tener principios estructurantes de la información.

- Capacidad para manejar y superar la ignorancia, entender y valorar lo diferente y diverso y soportarla incertidumbre y cambiar perspectivas.

o. La educación a través de las TIC puede constituirse en una respuesta a la complejidad de los cambias sociales, económicos y técnico-organizativos, que inciden en el mundo del trabajo, en la formación, en la educación permanente y que implica aprendizaje autodirigido. Los desarrollos de la ciencia cognitiva en la motivación y el aprendizaje ofrecen nuevas posibilidades para describir conceptualmente formas abiertas de acción, de aprendizaje y de trabajo.

d. Aprender, conocer no es sólo acceder a la información sino, como dice Sancho (95: 67), entrar en diálogo con ella, interrogarla y darle sentido (poner en juego la inteligencia colectiva). No es suficiente "navegar" en el océano de información sino aprender a darle 
estructura y significada. Como dice Mann (1993: 67), "el problema universal para cualquier ciudadano es adquirir las informaciones Sobre el mundo y cómo adquirir la posibilidad de articularlas y organizarlas. Para ello se impone una reforma del pensamiento".

\section{ESPACIOS DE CONOCIMIENTO}

Un aspecto que requiere consideración en la llamada inteligencia colectiva es la idea de espacio de conocimiento. Este no corresponde a un lugar físico. Es una metáfora para designar la vida intelectual de la humanidad. El conocimiento constituye un espacio simbólico y la inteligencia colectiva reconstruye y construye información significativa sobre el mundo natural y social, comportamientos, signas y cosas que existen o pueden existir en una dinámica de participación en la sociedad.

El espacio del conocimiento se puede considerar cama el sitio de creación de discursos capaces de transformar la realidad. Este es un espacio en el cual es posible navegar y la inteligencia colectiva organiza la interacción de diversidad de semióticas. Hoy, los hipertextos, los hiperdocumentos los multimedia interactivos, la inteligencia artificial, sistemas expertos, las ideografías dinámicas, los métodos de simulación digital y los sistemas de información interactiva, exploran semióticas cambiantes. Así, el espacio del conocimiento recorre la historia, la pluralidad de los lenguajes, las culturas y los mundos.

\section{CONOCIMIENTO COMPARTIDO}

En las sociedades generalmente, la metáfora dominante es que alguien sabe y da la información. El conocimiento fluye desde el poseedor del saber hacia los que no saben. En contraste en las sociedades de la información, la metáfora es el ámbito de la inteligencia colectiva donde se comparte y elabora conocimiento. Esta metáfora implica:

\section{a. Grupos diversos de personas}

Las perspectivas y las experiencias son importantes para enriquecer la información de todas las personas. La participación de diversidad de grupos enriquece la comprensión entre los mismos y provee oportunidades para entender contextos múltiples y aportar a los mismos.

\section{b. Búsqueda autorregulada de información}

Las personas aprenden a regular sus actividades, asumir sus propias responsabilidades y monitorear sus logros.

Búsqueda autorregulada de información significa que una persona motivada es capaz de buscarla en forma autónoma y baja su propia responsabilidad; de organizarla, aplicarla, controlarla y valorarla, sea en colaboración con otros o individualmente. Los procesos estratégicos dirigidos a la información y el conocimiento elaborado, incluyen dos conceptos: estrategias y control de la acción. El modelo cognitivo se basa entonces en el control metacognitivo y motivacional. 


\section{c. Intercambio libre de conocimiento}

Es posible que en la historia de la humanidad, este sea un momento crucial para ampliar los horizontes culturales y sociales del conocimiento. Una razón de ello es la posibilidad de creación de oportunidades de acceso y producción de información sin que haya censura.

Las personas se comunican. Colocan en las redes sus ideas, investigaciones, empresas, productos. Hay, si vale el término, una gran oferta de posibilidades en diferentes ámbitos de acuerda con las necesidades y los intereses de los grupos a de los individuas en particular.

Los beneficios teóricos del intercambio libre del conocimiento son claros. Es necesaria hacer explícitos los diferentes medios de acceso y demanda y además presionar el uso estratégico del conocimiento.

\section{d. Construcción de sentido}

La inteligencia colectiva es una filosofía de interacción y estilo colaborativo. No es una técnica de aula de clase. En diferentes situaciones, donde la gente necesita información o puede procurarla, se requiere una actitud y unas medias para compartirla.

Esta forma de inteligencia social se define sobre todo par un proceso de ayuda donde la gente interactúa para conseguir algunas objetivos pero sobre toda para construir sentidos de la información.

Este aspecto, aunque más teórico todavía que otros, se refiere a una aproximación al análisis, el uso de formas libres de comprensión y emplea de la información y a la naturaleza social del conocimiento.

El proceso de interacción en las redes por ejemplo, conlleva a una transacción de sentido más que a una transmisión de información. La diferencia radica en que no se trata de conocimiento canónico sino de un movimiento de construcción colectiva que entrecruza en diferentes rutas ( $y$ no hay una autoridad que pasea el saber y defina quién lo recibe, cómo y cuándo).

El resultado puede ser una transición desde la comprensión subjetiva de la información hacia la construcción social tanto del conocimiento como del aprendizaje. Por supuesta, cada persona puede pertenecer a diferentes comunidades de conocimiento donde comparta vocabulario, historia, valores, convenciones e intereses.

\section{IMPLICACIONES TECNOLÓGICAS}

El desarrollo de una inteligencia colectiva requiere la existencia de agentes que posibiliten interactuar, acceder y potenciar el uso de información así coma las relaciones entre pares y consultar, si es del caso, a diferentes expertas en diferentes dominios incluso dispersas por el planeta.

Se crean por tanto espacios de colaboración y comunicación entre varios agentes que pueden tomar diferentes formas. En general se puede hablar de localización de pares y formas colaborativas de comunicación libre entre agentes. Cada agente pasee la libertad 
de decidir cuándo y quién puede ayudar. Cualquier información o necesidad de la misma contiene la identificación del agente. Este aspecto es necesario porque un agente puede recibir y enviar múltiples respuestas.

Con el desarrollo de nuevas vías de acceso y procesamiento de la información (navegación individual, flujo continuo de información, simulaciones, alto poder de procesamiento) y la desterritorialización de las bibliotecas y bases de conocimiento, las nuevas tecnologías de la información y la comunicación transforman la relación entre conocimiento y aprendizaje. También hoy se desarrollan investigaciones con la participación de instituciones lejanas entre si y se llevan a cabo proyectos en laboratorios virtuales lo cual permite mayor eficiencia y cooperación nacional e internacional ${ }^{2}$.

\section{ESTRATEGIAS Y DISPOSITIVOS}

El desarrollo de una inteligencia colectiva conlleva que las comunidades humanas se comuniquen y reflexionen entre sí, envueltas en una negociación permanente de relaciones y contextos compartidos. Estos mundos requieren recursos, ambientes y conexiones cosmopolitas entre comportamientos y cosas inmersas en varios microcosmos, tecnología y maquinaria social que cruza jos diferentes senderos de la interacción. En esta interacción humana resulta de desarrollos, descubrimientos, y usos diferentes y reiterados.

Lo anterior por tanto, implica el análisis de algunas estrategias y dispositivos que posibiliten la navegación por el conocimiento y potencien su crecimiento. Conviene entonces abrir la mente hacia formas hipertextuales, a la teoría de la acción y las redes, a la teoría de la narratividad a través de modelamiento, la simulación y desarrollo de escenarios y a la teoría del arte en relación con la inteligencia artificial.

Por otra parte, el empleo de las redes de información (Internet, Intranet) es una condición de posibilidad muy grande para el desarrollo de inteligencias colectivas. Estas redes abren oportunidades de desarrollo personal y de comunicación a escala mundial. Las redes facilitan las relaciones interpersonales, el trabajo cooperativo y permiten compartir y debatir ideas. Las redes integran enorme cantidad de información multimedial de todo tipo y sobre cualquier tópico y proporciona un ambiente de acceso a numerosos programas y entornas lúdicos que facilitan el aprendizaje autónomo.

Las redes poseen modalidades de comunicación como el correo electrónico (e-mail), las listas de discusión, los grupos de noticias, los grupos de conversación (internet Relay Chat), las videocomunicaciones, las páginas web (escritas en HTML, Hiperlext Markup Language, aunque ya se puede utilizar un editor sencillo de textos word o netscape).

En general se pueden mencionar las siguientes estrategias que constituyen entonces oportunidades para el desarrollo de la IC:

- De aprendizaje cooperativo y círculos telemáticos.

- Entornas de aprendizaje (con características de interactividad, ramificación, transparencia y navegabilidad).

\footnotetext{
${ }^{2}$ Ver en este sentido el programa de Aplicaciones Telemáticas (continuación del DELTA) que cubre 30 proyectos de capacitación en la Unión Europea, el programa Sócrates para promover la mejora global de la educación y el programa Leonardo creado para fomentar el desarrollo de la capacitación profesional en Europa.
} 
- Multimediales.

- Materiales computarizados apropiados (material de autoenseñanza).

- Emplea intensiva de nuevos medios (correo electrónico, www, front page).

- Telebibliotecas y bibliotecas electrónicas.

- Recursos didácticos on-line.

- Centro de recursos Web: personales, empresariales, académicas, sociales, tiendas virtuales, Webs temáticas.

- Entornas tutorizadas de teleinformación y asesoramiento.

- Prensa electrónica.

Finalmente, estos recursos y estrategias para estimular el desarrollo de inteligencias colectivas pueden poseer al menos estas características:

a. Facilidad de acceso y empleo: que las personas que naveguen puedan hacerlo fácilmente, localizar la información, obtener materiales, encontrar enlaces y compartir dudas.

b. Calidad de entorno audiovisual: diseño claro y atractivo.

c. Calidad técnica y estética: opciones, iconos, ventanas, botones, espacios de textoimagen, elementos multimediales, estilo y lenguaje apropiado.

d. Calidad de contenidos: Información bien estructurada, amplia, sin censura.

e. Sistemas de navegación e interacción: entornas transparentes que permitan al usuario tener el control. Es decir, que permitan la navegación libre.

f. Bidireccionalidad: las personas reciben y a su vez producen información.

g. Potencialidad comunicativa: los espacias deben abrir canales comunicativas mediante enlaces y otras medias.

\section{CONCLUSIÓN}

Hasta ahora este asunto de desarrollar inteligencia colectiva comienza. Es un reto contemporáneo el de impulsar de nueva la autonomía y el protagonismo de los seres humanas frente a la generación de saberes y al manejo de tanta información. La interconectividad es una condición de pasibilidad para crear comunidades más pensantes, que potencien sus capacidades de manera más equitativa. La construcción de esta inteligencia resulta como dice, Klimovsky (1995:8), muy atractiva para aprender a dialogar coma navegantes y cartógrafos del ciberespacro.

\section{BIBLIOGRAFÍA CONSULTADA}

Cabero, Julio, et al. "Evaluación de medios y materiales de enseñanza en soporte multimedia". En: Pixel- BIT Revista de Medios y Educación. Secretariado de Recursos Audiovisuales y Nuevas Tecnologías. N 13-julio de 1999.URL.

HYPERLINK http://www.us.es/sav.http:// www.us.es/sav. 
EDUTEC. Nuevas tecnologías en la formación flexible y a distancia. Sevilla: Universidad de Sevilla, 1999.

Klimovsky, Gregorio. 'Enseñanza de la tecnología'. En: Teleconferencias y Aprendizaje a Distancia. California: ABC, California, USA, 1997.

Lévy, Pierre. "Cibercultura y educación". En: Conferencia sobre La Universidad en la Sociedad de la Información. Sao Paulo, 23 a 25 de octubre de 1996.

Lévy, Pierre. Collective intelligence. New York: Ed. Robert Bononno, 1997.

Loing, Bernard. "Educación y tecnología de la información. Cómo cerrar la brecha". En: Conferencia sobre La Universidad en la Sociedad de la Información. Sao Paulo, 23 a 25 de octubre de 1996.

Maldonado, Luis. Proyecto TECNICE. Una retrospectiva de diez años. Bogotá: Universidad Pedagógica Nacional, 1999.

Morin, Édgar. El desafío de la globalidad. Archipiélago,16, 1993.

PIXEL-BIT. Revista de medios y educación. Secretariado de Recursos Audiovisuales y Nuevas Tecnologías. N ${ }^{\circ} 13$, julio de 1999. URL: HYPERLINK http:// www.us.es/sav http://www.us.es/sav.

Ouéau, Philippe. Lo virtual. Barcelona: Paidós, 1995

Sancho, Juana Ma. "Educación y saciedad industrial: a vueltas con las paradojas" En: Hoy ya es mañana. Tecnologías y Educación: Un diálogo necesario. Comp. Juana Ma. Sancho y Luis Miguel Millán. Publicaciones M.C.E.P. Sevilla, 1995.

Scheunpflug, Annette. "La globalizacián como desafío al aprendizaje humano". En: Educación. Tübingen, 1997.

Straka, Gerald et al. "Un modelo de aprendizaje motivado y autodirigido". En: Educación. Tübingen, 1997

Tye, K. Global education: From thought fo action. Alejandría (VA), Association and Curriculum Development, 1991. 